\title{
Spatial coherence of forward-scattered light in a turbid medium
}

\author{
Changhuei Yang, Kyungwon An,* Lev T. Perelman, Ramachandra R. Dasari, and Michael S. Feld \\ George R. Harrison Spectroscopy Laboratory, Massachusetts Institute of Technology, 77 Massachusetts Avenue, \\ Cambridge, Massachusetts 02139
}

Received June 1, 1998; revised manuscript received November 18, 1998; accepted November 25, 1998

\begin{abstract}
We study spatially coherent forward-scattered light propagating in a turbid medium of moderate optical depth (0-9 mean free paths). Coherent detection was achieved by using a tilted heterodyne geometry, which desensitizes coherent detection of the attenuated incident light. We show that the degree of spatial coherence is significantly higher for light scattered only once in comparison with that for multiply scattered light and that it approaches a small constant value for large numbers of scattering events. (C) 1999 Optical Society of America [S0740-3232(99)01104-7]
\end{abstract}

OCIS codes: $030.1640,290.4210,290.7050,040.2840$.

\section{INTRODUCTION}

Coherence in elastically scattered light can give rise to interesting and potentially useful physical phenomena such as coherent backscatter ${ }^{1}$ and laser oscillation in turbid media. $^{2}$ Useful information about the turbid medium can be extracted through coherent measurement of the Wigner phase space. ${ }^{3}$ In addition, optical coherence tomography studies have demonstrated that coherent scattering from tissue interfaces is useful for near-surface tissue imaging. ${ }^{4}$ In light of these developments, it is important to further our understanding of the temporal and spatial coherence of scattered light in relation to the number of scattering events that it undergoes. This can lead to techniques for selective detection of short transmission paths through measurement of the extent of coherent scattering. Such a method would complement intensity-based time gating techniques, ${ }^{5,6}$ polarization loss methods, ${ }^{7,8}$ and other techniques under study using optical photons for biomedical imaging.

Although research in the field of biomedical imaging is currently dominated by intensity-based techniques, the use of coherence-based techniques offers some interesting potential opportunities. Whereas intensity-based techniques are sensitive only to the field amplitude of the propagating wave, coherent-based techniques sample the phase front as well. From a fundamental point of view, both the amplitude and the phase front of a wave propagating through a scattering medium experience degradation with increasing numbers of scattering events. Although much theoretical work has been devoted to studying intensity-based processes, relatively little fundamental work has been done to model the behavior of coherence-based processes.

As a first step, we simultaneously study the decay of intensity-based and coherent-based signals in a scattering medium. We find that it is possible to categorize the scattered light according to the number of scattering events $j$ that each has undergone. In addition, we introduce the quantity $a_{j}$ to parameterize the phase-front uni- formity of the scattered light that has undergone exactly $j$ scattering events. By comparing the intensity-based and coherence-based signals, we obtain experimental values for the $a_{j}$ 's. Our experiments, which study moderatescattering samples [up to 9 mean free paths (MFP's)], combined with a related study of coherent detection in optically thick scattering media (20-50 MFP's), ${ }^{9}$ show that $a_{j}$ decreases rapidly for small $j$ 's and that it reaches a constant value for large $j$ 's. This conclusion provides interesting insight into the picture of wave propagation in a scattering medium and how research into coherencebased biomedical imaging may proceed.

\section{FORMULATION}

In the literature of light scattering in a turbid medium, ${ }^{10,11}$ the term coherent intensity is often used to refer to the attenuated incident light transmitted through the medium, whereas incoherent intensity refers to light scattered in other directions (also called diffusive light). The term coherence can be used in two contexts, to describe either temporal or spatial coherence. Spatial coherence is a measure of the electromagnetic field's phasefront uniformity and is the subject of the present study. As shown below, the incoherent intensity preserves a significant amount of spatial coherence. To avoid possible confusion, we shall refer to coherent intensity as unscattered light and incoherent intensity as scattered light. Note that the coherent intensity can be formally described by the destructive interference of the incident field with the fields from the scatterers.

As with most coherent detection experiments, ${ }^{4,9,12}$ we measure the transmission by means of a heterodyne technique. A portion of a laser beam shifted in frequency by $\Delta \omega$ is sent through a turbid medium. The electric field of the exiting beam reaching the detector can be expressed as 


$$
\begin{aligned}
E_{T}(t) \exp \left[i \phi_{T}(\boldsymbol{\rho})\right]= & \left\{\sum_{j=0}^{\infty} E_{j} \exp \left[i \phi_{j}(\boldsymbol{\rho})\right]\right\} \\
& \times \exp [i(\omega+\Delta \omega) t],
\end{aligned}
$$

where $E_{T}(t)$ is the (real) field amplitude and $\phi_{T}(\boldsymbol{\rho})$ is the phase front, with $\rho$ being the spatial coordinate at the surface of the detector. In this expression $E_{j}$ and $\phi_{j}(\boldsymbol{\rho})$ are the net field amplitude and phase that are due to all components of the transmitted wave scattered exactly $j$ times within the turbid medium; $E_{j}$ can be considered constant over the small area of our detector. It is to be understood that $E_{0}$ and $\phi_{0}(\boldsymbol{\rho})$ are the electric-field amplitude and phase associated with unscattered light. $E_{j}$ and $\phi_{j}(\boldsymbol{\rho})$ are given by the sum over all field trajectories (i.e., rays in the geometrical-optics sense) with exactly $j$ scattering events:

$$
E_{j} \exp \left[i \phi_{j}(\boldsymbol{\rho})\right]=\sum_{\text {traj }} E_{j, \text { traj }} \exp \left[i \phi_{j, \text { traj }}(\boldsymbol{\rho})\right],
$$

where $E_{j \text {,traj }}$ is the electric field associated with a specific trajectory with exactly $j$ scattering events in the medium and $\phi_{j, \text { traj }}(\boldsymbol{\rho})$ is the associated phase. For readers conversant with scattering theory, the field associated with the $j$ th scattering event and the corresponding field trajectories are equivalent to the $j$ th integral and scattering processes, respectively, of Twersky's integral equation. ${ }^{11}$ However, the solution of this equation is not trivial, and in this paper we adopt a different approach in which propagation of fields between scatterers is treated in the geometrical-optics limit.

The transmitted light is recombined with the unshifted component of the beam at the detector. The resulting modulated heterodyne signal can be expressed as

$\operatorname{Re}\left\{2 E_{r}^{*}(t) E_{T}(t) \exp \left[i \phi_{T}(\boldsymbol{\rho})\right]\right\}$

$$
=\sum_{j=0}^{\infty} E_{r} E_{j}\left\{2 \cos \left[\Delta \omega t+\phi_{j}(\boldsymbol{\rho})\right]\right\},
$$

where $E_{r}$ is the amplitude of the reference beam and \{\} denotes averaging over the detector area.

We can recast the spatial averaged quantities on the right-hand side of Eq. (3) as

$$
\left\{2 \cos \left[\Delta \omega t+\phi_{j}(\boldsymbol{\rho})\right]\right\}=a_{j} \cos \left(\Delta \omega t+\bar{\phi}_{j}\right),
$$

where $a_{j}$, the spatial coherence factor, is a measure of the uniformity of $\phi_{j}(\boldsymbol{\rho})$ and $\bar{\phi}_{j}$ is the effective phase shift upon spatial averaging. In other words, from the heterodyne signal amplitude, we can parameterize the uniformity of the transmitted light phase front through the $a_{j}$ 's. The amplitude of the heterodyne signal can then be expressed as

$$
\begin{aligned}
& \left|\operatorname{Re}\left\{2 E_{r}^{*}(t) E_{T}(t) \exp \left[i \phi_{T}(\boldsymbol{\rho})\right]\right\}\right|_{\mathrm{amp}} \\
& \quad=E_{r}\left[\sum_{j=0}^{\infty}\left(a_{j} E_{j}\right)^{2}+\sum_{i=0}^{\infty} \sum_{\substack{j=0 \\
j \neq i}}^{\infty} a_{i} a_{j} E_{i} E_{j} \cos \left(\bar{\phi}_{i}-\bar{\phi}_{j}\right)\right]^{1 / 2} \\
& \quad \approx E_{r}\left[\sum_{j=0}^{\infty}\left(a_{j} E_{j}\right)^{2}\right]^{1 / 2} .
\end{aligned}
$$

We assume that the $\bar{\phi}_{j}$ 's are uncorrelated, so that upon an averaging over a sufficient number of measurements, the cross terms in Eq. (5) are negligible. The above assumption may be justified by noting that field trajectories that contribute to $\phi_{j}(\boldsymbol{\rho})$ and consequently to $\bar{\phi}_{j}$, as expressed in Eq. (2), do not have any phase correlation with field trajectories associated with $\phi_{i}(\boldsymbol{\rho})$, where $j \neq i$. This lack of phase correlation can be illustrated by considering two trajectories that follow the same path, except that one of them (trajectory A) undergoes one more scattering event at the end than the other (trajectory B). Even this pair of trajectories, which are so similar to each other by reason of their nearly identical paths, will not have any phase correlation. This is because the extra path length that trajectory A has by reason of the additional scattering will be approximately 1 mean-free-path length, which is approximately $2 \mathrm{~mm}$ long in a typical experiment; since this length is orders of magnitude larger than the optical wavelength, the two trajectories would not have any phase correlation. The lack of phase correlation in the constituent field trajectories associated with different numbers of scattering events implies that the $\phi_{j}(\boldsymbol{\rho})$ 's, and consequently the $\bar{\phi}_{j}$ 's, are uncorrelated with each other.

The transmitted intensity can be expressed as

$$
\begin{aligned}
T & =\left\{E_{T}^{*}(t) \exp \left[-i \phi_{T}(\boldsymbol{\rho})\right] E_{T}(t) \exp \left[i \phi_{T}(\boldsymbol{\rho})\right]\right\} \\
& =\sum_{j=0}^{\infty} E_{j}{ }^{2}+\sum_{i=0}^{\infty} \sum_{\substack{j=0 \\
j \neq i}}^{\infty} E_{i} E_{j}\left\{2 \operatorname{Re}\left(\cos \left[\phi_{i}(\boldsymbol{\rho})-\phi_{j}(\boldsymbol{\rho})\right]\right)\right\} \\
& \approx \sum_{j=0}^{\infty} E_{j}{ }^{2} .
\end{aligned}
$$

Again, we have assumed that the $\phi_{j}(\boldsymbol{\rho})$ 's are uncorrelated and that the terms $\left\{2 \operatorname{Re}\left(\cos \left[\phi_{i}(\boldsymbol{\rho})-\phi_{j}(\boldsymbol{\rho})\right]\right)\right\}$ are sufficiently small that the cross terms are negligible.

In an experiment in which the reference beam is aligned to be parallel with the unscattered beam, $a_{0}$ will be close to its maximum value of 2 . This is because the planar wave front of the unscattered component is preserved, resulting in a uniform phase front. On the other hand, the scattered component will likely have an irregular wave front, leading to smaller values of the $a_{j}$ 's for $j$ $\neq 0$. For a turbid medium of moderate optical depth, the heterodyne signal is dominated by the unscattered component, as both $a_{0}$ and $E_{0}{ }^{2}$ are large. The scatteredlight contribution becomes significant only when $E_{0}{ }^{2}$ is substantially attenuated. Since $a_{0}$ is much larger than the rest of the spatial coherence factors, this transition requires an optically thick turbid medium. However, in this regime, light scattered only a few times is negligible in comparison with light scattered multiple times. In other words, there is no situation where the coherent contribution from light scattered a few times is significant.

Fortunately, there is a way to circumvent this difficulty. If we can reduce $a_{0}$ with respect to the rest of the $a_{j}$ 's and decrease $E_{0}{ }^{2}$ at the same time, the contribution of the scattered beam to the heterodyne signal can be made significant at lower optical depths. This can be achieved by tilting the unscattered beam traversing the sample by a small angle relative to the reference beam, as 


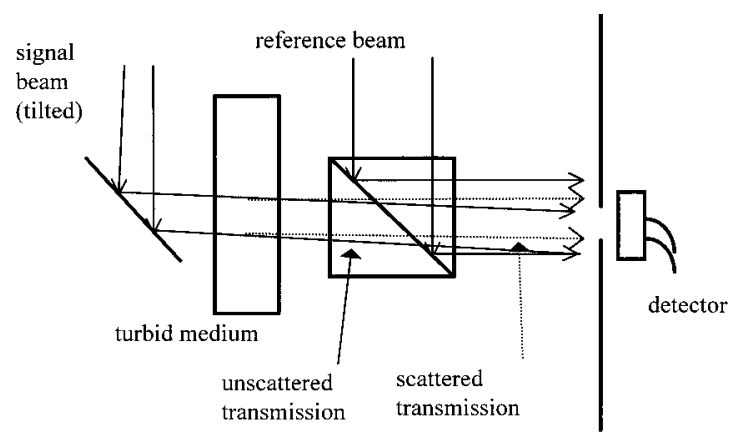

Fig. 1. Tilted heterodyne geometry, schematic diagram.

shown in Fig. 1. The tilt will confer a linearly varying $\phi_{0}(\boldsymbol{\rho})$ across the detector and significantly reduce $a_{0}$. On the other hand, the rest of the $a_{j}$ 's will experience little of this effect because the scattered light that reaches the detector will still be approximately parallel to the reference beam. Moreover, the tilt will also reduce the unscattered component that reaches the detector because the unscattered beam will be off center from the detector. In contrast, the scattered transmission will not be much affected, as it subtends a wider solid angle.

\section{EXPERIMENTS}

Our experiments employ the above technique to study the spatial coherence of scattered light in turbid media with varying optical depth. The optical depth $D$ is measured as the number of scattering MFP's and is equal to the average number of scattering events undergone by the incident light in the medium. In our experiments $D$ ranges from 0 to 9 . We used a cw Ti:sapphire laser (Coherent MIRA 600) operating at $753 \mathrm{~nm}$ with a beam width of 1.5 $\mathrm{mm}$ and an angular divergence of $1.2 \mathrm{mrad}$. The beam was split into a frequency-shifted signal beam and an unshifted reference beam. The frequency of the signal beam was shifted by a pair of acousto-optic modulators (ISOMET 1206-C) driven by a dual-frequency driver (IntraAction DFE-1102A4) at approximately $110 \mathrm{MHz}$, with the two frequencies offset by approximately $70 \mathrm{kHz}$. The geometry was such that the acousto-optic modulators shifted the modulated beam in opposite directions, producing a net shift of $70 \mathrm{kHz}$ relative to the reference beam. The signal beam was sent through a turbid medium consisting of polystyrene microspheres (Polysciences) of diameter $d=4.329 \mu \mathrm{m}$ and refractive index $n=1.59$ in a solution of distilled water. The transmitted beam was then recombined with the reference beam and focused on a silicon photodiode detector (EG\&G FND$100)$ that subtended a solid angle of $3.5 \times 10^{-6} \mathrm{sr}$ from the turbid medium. The detection area was determined by an aperture of $560-\mu \mathrm{m}$ diameter. The reference beam power impinging on the detector was typically $0.65 \mathrm{~mW}$, and the signal beam, transmitted through clear water, was approximately the same. With the acousto-optic modulator driver used as a reference, the heterodyne signal amplitude could be measured very accurately with a dual-phase lock-in amplifier (Stanford Research SR830) operating with a detection bandwidth of $10 \mathrm{~Hz}$.
For each experiment calibration was first done with clear water in the sample cell. By measuring the heterodyne signal amplitude and the transmitted intensity, which equals $E_{0}{ }^{2}$ in the absence of scatterers, one can compute $a_{0}$. The turbid medium was then introduced and diluted gradually. At each value the heterodyne signal amplitude and the total transmitted intensity were recorded.

The product of the turbid medium concentration, the computed Mie scattering cross section, ${ }^{13}$ and the transmission path length gives the number of MFP's, which is the exponential attenuation factor for transmission of unscattered light. We define the quantity $S$ as a measure of detected coherence:

$$
\begin{aligned}
S & =\frac{\left|\operatorname{Re}\left\{2 E_{r}^{*}(t) E_{T}(t) \exp \left[i \phi_{T}(\boldsymbol{\rho})\right]\right\}\right|_{\mathrm{amp}}^{2}}{a_{0}{ }^{2} E_{r}{ }^{2}} \\
& =E_{0}{ }^{2}+\sum_{j=1}^{\infty}\left(\frac{a_{j}}{a_{0}}\right)^{2} E_{j}{ }^{2} .
\end{aligned}
$$

As can be seen in Eq. (7), $S$ approximates $E_{0}{ }^{2}$ when scattered transmission does not contribute significantly to the heterodyne signal. Any deviation of $S$ from $E_{0}{ }^{2}$ therefore indicates a significant coherent contribution from the scattered transmission.

Figures 2(a) and 2(b) plot, respectively, the total transmitted intensity $T$ and $S$ for various angular tilts of the transmitted beam. The curves are all normalized to unity at $D=0$. It can be seen from Fig. 2(a) that the decline in the transmitted intensity is more gradual for larger tilt angles. ${ }^{14}$ This indicates the presence of a growing contribution from scattering with angular tilt. In addition, for zero angular tilt the attenuated total intensity is approximately exponential. In a previous study, the slope of this curve was found to decrease at larger $D$ (from 20 to $50 \mathrm{MFP}$ 's). ${ }^{5}$ This implies that the slope of the zero angular tilt transmitted-intensity curve is mainly due to the attenuated unscattered component over the range of $D$ shown in Fig. 2(a) and that the contribution to this curve from scattering is negligible until larger values of $D$ are reached. With this interpretation an important result is evident in Fig. 2(b). The $S$ profiles for all angular tilt above $0 \mathrm{mrad}$ are above that for zero angular tilt, which simply exhibits the coherent attenuation of unscattered light. This implies from Eq. (7) that scattered light contributes to the observed coherent signal. Moreover, this deviation from coherent attenuation of unscattered light occurs at values of $D$ as small as unity; the component of scattered light that causes this deviation can have been scattered only a few times.

In the experiments we observed a slow fluctuation in the heterodyne signal amplitude over a time scale of $0.1-1 \mathrm{~s}$. This suggests that the wave front of the scattered contribution fluctuates in time. The fluctuation of the wave front can change its uniformity over time, which leads to changing $a_{j}$ 's, where $j \neq 0$. This wave-front fluctuation is likely caused by Brownian motion drifts of the microspheres, which slowly alter the phase of each possible path among the microspheres. The calculated rms 


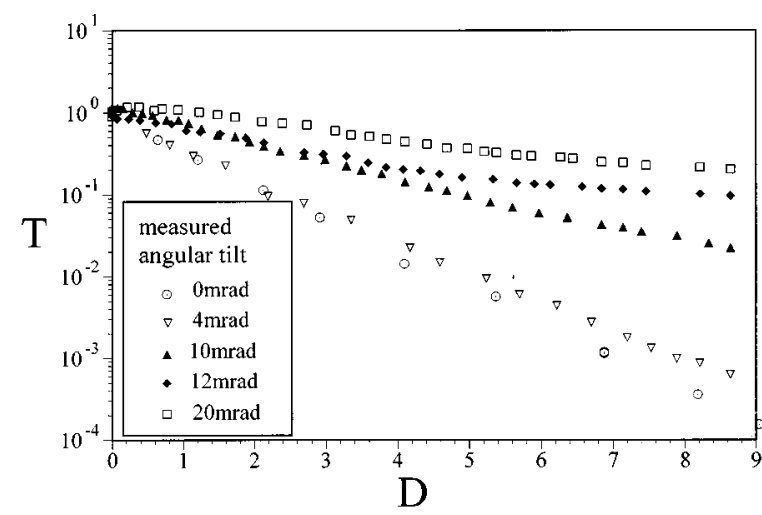

(a)

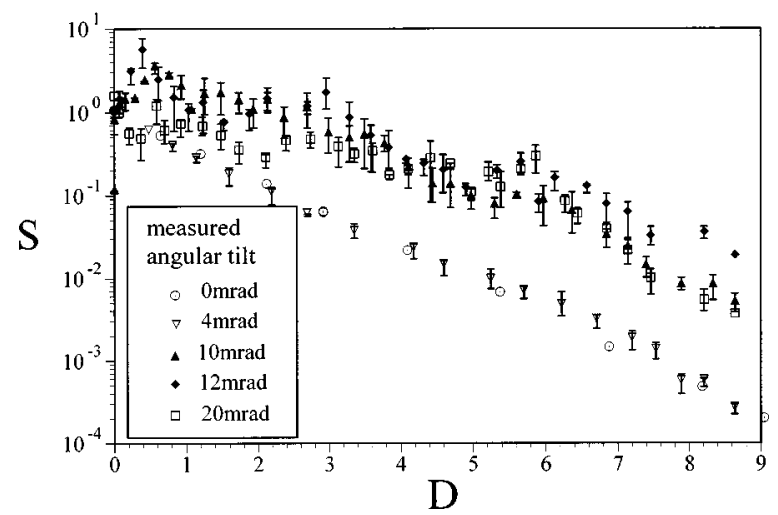

(b)

Fig. 2. (a) Total transmitted intensity versus number of MFPs, $D$, for various tilt angles. (b) $S$, computed from Eq. (4), versus number of MFPs, $D$, for various tilt angles; error bars denote the extent of fluctuations in the heterodyne signals.

displacement of a microsphere in water in $1 \mathrm{~s}$ is approximately $1 \mu \mathrm{m}$, which is comparable with the wavelength of the light. ${ }^{15}$

Finally, it is important to understand that the coherent detection of scattered light is caused not only by the reduction of $E_{0}{ }^{2}$ that is due to the off-centered transmission. The relative reduction of $a_{0}$ with respect to the rest of the $a_{j}$ 's in the tilted geometry is also important. In a similar set of experiments done with a laterally displaced signal beam, which provided similar $E_{0}{ }^{2}$ reduction but without similar $a_{0}$ change, scattered light could not be observed in the coherently detected signal.

\section{THEORETICAL MODEL}

As seen in Fig. 2(b), aside from the initial change in slope for small $D$, the slopes of $S$ for all tilt angles match that of the curve for zero angular tilt, which describes the attenuation of the unscattered beam. More importantly, the profiles of $S$ for all tilt angles above $0^{\circ}$ do not match those of the associated transmitted intensities $(T)$. Since $S$ differs from $T$ in that it is a function of the phase wavefront uniformity parameters $a_{j}$ [Eq. (7)], this suggests that the $a_{j}$ 's vary over the range of $j$. In other words, the phase-front uniformity of that particular component of scattered light depends on the number of scattering events that it has undergone.
The values of the $a_{j}$ 's can be determined by fitting the experimental data in Fig. 2(b) if the $E_{j}{ }^{2}$ quantities are known. With this motivation we formulate the following theoretical model, which gives an expression for $E_{j}{ }^{2}$ for low $j$ values as a function of tilt angle.

Consider a turbid medium of optical thickness $D$; the distance along the $z$ axis is measured in units of $D$ (Fig. 3 ). The relation of $E_{j}$ to $D$ can be derived by means of a three-dimensional model of scattering. The following equation is obeyed by the scattered light traversing an incrementally small slice $\delta D^{\prime}$ of the medium located at a distance $D^{\prime}$ :

$$
\begin{aligned}
\delta\left(E_{j}^{2}(D, \boldsymbol{\vartheta})\right)= & E_{j-1}^{2}\left(D^{\prime}, \boldsymbol{\vartheta}^{\prime}\right) \\
& \times\left[P_{S}\left(f\left(\boldsymbol{\vartheta}, \boldsymbol{\vartheta}^{\prime}\right)\right) \delta D^{\prime}\right] \exp \left\{-\frac{D-D^{\prime}}{\cos [f(\boldsymbol{\vartheta}, \mathbf{z})]}\right\} .
\end{aligned}
$$

Here $E_{j}{ }^{2}(D, \boldsymbol{\vartheta})$ denotes the intensity of light arriving at distance $D$ in direction $\boldsymbol{\vartheta}$ after undergoing exactly $j$ scattering events, $P_{S}(\theta)$ is the phase function, i.e., the distribution function that governs the directional change of light in the course of a scattering event, and $f(\boldsymbol{\vartheta}, \boldsymbol{\nu})$ is the angle between $\boldsymbol{\vartheta}$ and $\boldsymbol{\nu}$. The first factor on the righthand side of Eq. (8) represents the intensity of light that has been scattered $j-1$ times before entering the slice in direction $\boldsymbol{\vartheta}^{\prime}$. The second factor represents the probability that this input light is scattered exactly once within the slice in direction $\boldsymbol{\vartheta}$. The last factor accounts for the attenuation of emerging light as it propagates unscattered through the remaining thickness of the scattering medium.

Since light that has undergone a few scattering events by the microspheres is highly forward directed, we can approximate $\boldsymbol{\vartheta}$ by the parameters $\theta_{x}$ and $\theta_{y} . \quad \theta_{x}$ is the angle between the $z$ axis and the projection of $\boldsymbol{\vartheta}$ on the $x-z$ plane, and $\theta_{y}$ is the corresponding parameter for the $y-z$ plane. Moreover, we can approximate the cosine in the last term by unity because we are considering only highly forward-scattered light. Finally, we approximate $P_{S}(\theta)$ by a Gaussian function $(k / \pi) \exp \left[-k\left(\theta_{x}^{2}+\theta_{y}^{2}\right)\right]$, with $k=135 \mathrm{rad}^{-2}$ for the microspheres used. The value of $k$ was established by computing $P_{S}(\theta)$ from Mie theory and fitting the result with a Gaussian function.

Integrating Eq. (8) over $D^{\prime}, \theta_{x}^{\prime}$, and $\theta_{y}^{\prime}$, we obtain

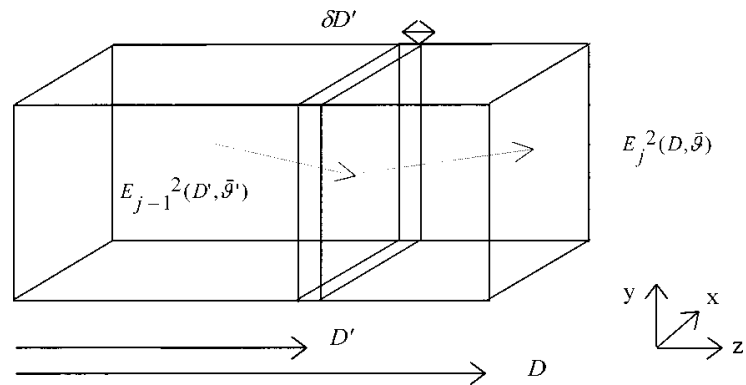

Fig. 3. Geometry used in the theoretical model. The distance along the $z$ axis is measured in units of the optical depth $D$. 


$$
\begin{aligned}
E_{j}^{2}\left(D, \theta_{x},\right. & \left.\theta_{y}\right) \\
\approx & \int_{0}^{D} \int_{-\pi / 2}^{\pi / 2} \int_{-\pi / 2}^{\pi / 2} E_{j-1}^{2}\left(D^{\prime}, \theta_{x}^{\prime}, \theta_{y}^{\prime}\right) \\
& \times\left(\frac{k}{\pi} \exp \left\{-k\left[\left(\theta_{x}-\theta_{x}^{\prime}\right)^{2}+\left(\theta_{y}-\theta_{y}^{\prime}\right)^{2}\right]\right\}\right) \\
& \times \exp \left[-\left(D-D^{\prime}\right)\right] \mathrm{d} \theta_{x}^{\prime} \mathrm{d} \theta_{y}^{\prime} \mathrm{d} D^{\prime} \\
\approx & \int_{0}^{D} \int_{-\infty}^{\infty} \int_{-\infty}^{\infty} E_{j-1}{ }^{2}\left(D^{\prime}, \theta_{x}^{\prime}, \theta_{y}^{\prime}\right) \\
& \left.\times\left[\frac{k}{\pi} \exp \left(-k\left[\left(\theta_{x}-\theta_{x}^{\prime}\right)^{2}\right]+\left(\theta_{y}-\theta_{y}^{\prime}\right)^{2}\right]\right)\right] \\
& \times \exp \left[-\left(D-D^{\prime}\right)\right] \mathrm{d} \theta_{x}^{\prime} \mathrm{d} \theta_{y}^{\prime} \mathrm{d} D^{\prime} .
\end{aligned}
$$

We approximate the profile of the input beam by a Gaussian function

$$
E_{0}{ }^{2}\left(D=0, \theta_{x}, \theta_{y}\right)=E_{\text {input }}^{2} \exp \left[-\alpha\left(\theta_{x}{ }^{2}+\theta_{y}{ }^{2}\right)\right],
$$

where $E_{\text {input }}$ is the electric-field amplitude of the beam in the forward direction before entering the turbid medium.

It can be proven by induction that the solution to relation (9) given the input as expressed in Eq. (10) is

$$
\begin{aligned}
E_{j}^{2}\left(D, \theta_{x}, \theta_{y}\right) \approx & E_{\text {input }}^{2}\left\{\left(\frac{k}{j \alpha+k}\right)\right. \\
& \left.\times \exp \left[-\left(\frac{k \alpha}{j \alpha+k}\right)\left(\theta_{x}^{2}+\theta_{y}{ }^{2}\right)\right]\right\} \\
& \times\left[\frac{D^{j}}{j !} \exp (-D)\right] .
\end{aligned}
$$

As mentioned above, this result is valid only for light scattered a few times, so that the light is confined within a narrow cone in the forward direction. The $E_{j}{ }^{2}$ 's for large $j$ 's are more diffusive, and the above small-angle approximation will no longer hold. For small values of $D$, the total transmitted intensity given by Eq. (6) is dominated by the $E_{j}{ }^{2}$ terms, where the $j$ 's are small. As such, we expect that Eqs. (6) and (11) will give a good fit to the experimental curves in Fig. 2(a) for small values of $D$. Since the theoretical model overestimates the $E_{j}{ }^{2}$ 's for large $j$ 's, we expect the theoretical fits to deviate upward from the experimental curves at large values of $D$.

\section{APPLICATION OF THE MODEL AND DISCUSSION}

To verify the validity of the model, we applied it to the transmitted-intensity experimental data of Fig. 2(a). In our case the input laser beam has a sharp Gaussian profile superposed on a broader tapering base. We approximated the beam as the summation of two Gaussian curves. The $\alpha$ parameter of the sharper Gaussian is approximately $1 \times 10^{5} \mathrm{rad}^{-2}$, and that for the broader Gaussian is approximately $1 \times 10^{3} \mathrm{rad}^{-2}$; the intensity of the second Gaussian is approximately $0.15 \%$ the intensity of the first Gaussian at the center of the beam. The results are shown in Fig. 4. As can be seen, the theory gives good fits to the experimental data for values of $D$ from 0 to 5 for various angular tilts. The fits are poorer for $D>5$, as expected. The theoretical curves are very sensitive to variations in the angular tilt; we found that good fits to some of the experimental data are given by angular tilts that are approximately $20 \%$ lower than experimentally measured values. This discrepancy is likely due to errors in the experimental determination of the angular tilts.

Having substantiated our model, we now employ it in Eq. (7) to extract values for the $a_{j}$ 's. We focus our attention on the experimental data for 10-mrad angular tilt (we use the fit value of $8 \mathrm{mrad}$ ), for which our signal-tonoise ratio is high. Two features can be noted in the experimental data (Fig. 5). First, there is a plateau at small $D$; this implies that the $a_{j} / a_{0}$ factors are relatively large for small $j$ 's. Second, the slope of the experimental curve becomes the same as that of the unscattered curve for large $D$, which indicates that the $a_{j} / a_{0}$ factors are relatively small for large $j$ 's. The theory was fitted to the data from 0 to 5 MFP's by multiplying $S$ by $\exp (D)$ and doing a least-squares fit to the resulting polynomial curve. The $j=1$ component was found to be dominant, with $\left(a_{1} / a_{0}\right)^{2}=8.0$; for light scattered twice, $\left(a_{2} / a_{0}\right)^{2}$ $=0.5$, and the remaining terms are negligible. We next set the coefficients of $\left(a_{j} / a_{0}\right)^{2}$ at zero for $j>1$ and plotted the theoretical result against the whole range. As can be seen from Fig. 5, such a model, which accounts for

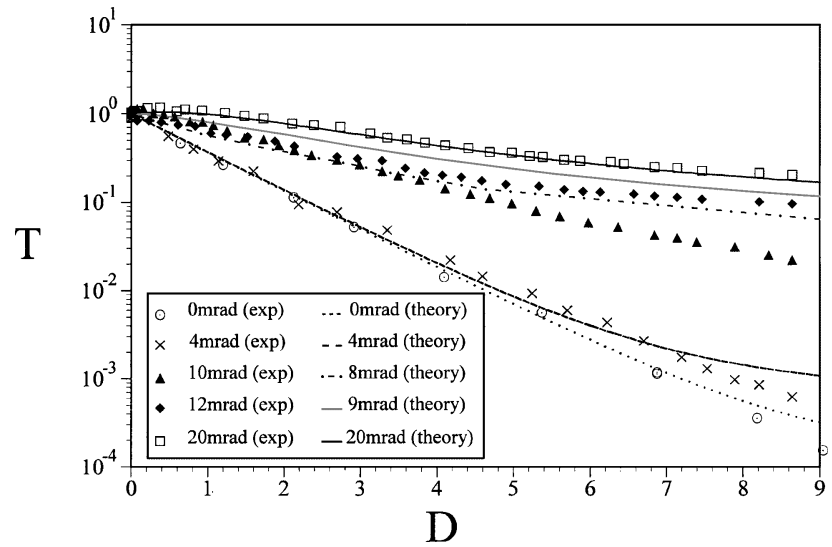

Fig. 4. Plot of transmitted intensity versus number of MFP's with theoretical fits.

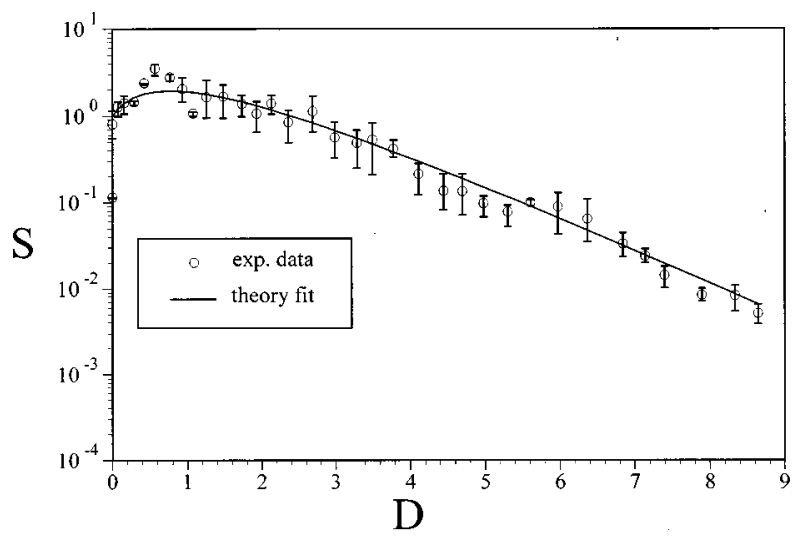

Fig. 5. Plot of $S$ versus number of MFP's for a tilt angle of 10 mrad with theoretical fit. The error bars denote the extent of fluctuations in the heterodyne signals. See text for details. 
the coherence of light scattered only once, gives a very good fit to the experiment. This further validates our assertion that the phase front of light scattered only once is significantly more uniform than the rest of the scattered light.

By examining the rest of the experimental data of Fig. $2(\mathrm{~b})$, we find that the decrease of $\left(a_{j} / a_{0}\right)^{2}$ becomes more gradual at larger angular tilts. [The small signal-tonoise ratio for the rest of the data prevents us from quantifying $\left(a_{j} / a_{0}\right)^{2}$ well.] By extending this observation of dependence on angular tilt, we can predict that, at an angular tilt of $0 \mathrm{mrad}$, we should see an even sharper decrease of $\left(a_{j} / a_{0}\right)^{2}$ than the results reported above for 10mrad angular tilt.

We can learn more about the spatial coherence of scattered light through the generalized model [Eq. (7)] by applying it to the results of a related heterodyne detection experiment ${ }^{9}$ performed in the regime of large MFP's (approximately 20-50). In that study there was no angular tilt in the detection scheme, so the coherent signal from the unscattered component dominated until sufficiently large values of MFP's were reached. That experiment employed a quantity equivalent to our parameter $S$. Not surprisingly, the dependence of $S$ was dominated by unscattered transmission for small values of $D$ (from 0 to $20)$. The curve then tapered off to a more gentle slope. From the provided data, ${ }^{9}$ we can infer that this latter slope matches the slope of the scattered-light intensity attenuation curve. The first fact indicates that the $\left(a_{j} / a_{0}\right)$ 's are much smaller than unity for that experiment, so that the coherent contribution from scattered light is insignificant in comparison with that from unscattered light until the intensity of unscattered light is attenuated sufficiently. The second fact indicates that the $\left(a_{j} / a_{0}\right)$ 's are approximately equal for large values of $j$, so that $S$ has the same slope as that of the transmittedintensity curve. In other words, Eq. (7) can be expressed for large $D$ as

$$
S \approx\left(\frac{a}{a_{0}}\right)^{2} \sum_{j=0}^{\infty} E_{j}{ }^{2} .
$$

This implies that the phase wave-front uniformity of light scattered a large number of times is relatively independent of the number of scattering events.

\section{CONCLUSION}

The picture of light scattering that emerges from this study raises some interesting questions. We find that the forward-scattered coherent phase front rapidly diminishes with the number of elastic scattering events, far more rapidly than the corresponding decrease in the intensity of the wave. However, a small but distinct coherent component persists, even for a large number of scattering events. It would be interesting to develop a theoretical model that can account for these changes in spatial coherence. It would also be interesting to explore the spatial distribution of the field trajectories in the medium. Such questions are of basic interest and have im- plications for coherent imaging as well. We hope that this paper stimulates further experiments and theory to answer such timely and interesting questions.

\section{ACKNOWLEDGMENTS}

The authors acknowledge valuable discussions with Jun $\mathrm{Wu}$ and Vadim Backman. This work was carried out at the MIT Laser Biomedical Research Center and was supported by National Institutes of Health grant P41RR02594 and a grant from Hamamatsu Corporation.

*Present address, Korea Advanced Institute of Science and Technology, 373-1, Kusong-dong, Yusong-gu, Taejon, 305-701, Korea.

Address correspondence to Changhuei Yang at the location on the title page or phone, 617-253-6203; fax, 617253-4513; e-mail, chyang@mit.edu.

\section{REFERENCES AND NOTES}

1. P. Wolf and G. Maret, "Weak localization and coherent backscattering of photons in disordered media," Phys. Rev. Lett. 55, 2696-2699 (1985).

2. W. L. Sha, C. Liu, and R. R. Alfano, "Spectral and temporal measurements of laser action of Rhodamine 640 dye in strongly scattering media," Opt. Lett. 19, 1922-1924 (1994).

3. A. Wax and J. E. Thomas, "Optical heterodyne imaging and Wigner phase space distributions," Opt. Lett. 21, 14271429 (1996).

4. J. A. Izatt, M. R. Hee, G. M. Owen, E. A. Swanson, and J. G. Fujimoto, "Optical coherence microscopy in scattering media," Opt. Lett. 19, 590-592 (1994).

5. K. M. Yoo and R. R. Alfano, "Time-resolved coherent and incoherent components of forward light scattering in random media," Opt. Lett. 15, 320-322 (1990).

6. L. T. Perelman, J. Wu, I. Itzkan, and M. S. Feld, "Photon migration in turbid media using path integrals," Phys. Rev. Lett. 72, 1341-1344 (1994).

7. J. M. Schmitt, A. H. Gandjbakhche, and R. F. Bonner, "Use of polarized light to discriminate short-path photons in a multiply scattering medium," Appl. Opt. 31, 6535-6546 (1992).

8. O. Emile, F. Bretenaker, and A. L. Floch, "Rotating polarization imaging in turbid media," Opt. Lett. 21, 1706-1708 (1996).

9. M. R. Hee, J. A. Izatt, J. M. Jacobson, J. G. Fujimoto, and E. Swanson, "Femtosecond transillumination optical coherence tomography," Opt. Lett. 18, 950-952 (1993).

10. A. Ishimaru, Wave Propagation and Scattering in Random Media (Academic, New York, 1978), Vol. 1.

11. A. Ishimaru, Wave Propagation and Scattering in Random Media (Academic, New York, 1978), Vol. 2.

12. M. Toida, M. Kondo, T. Ichimura, and H. Inaba, "Twodimensional coherent detection imaging in multiple scattering media based on the directional resolution capability of the optical heterodyne method," Appl. Phys. B 52, 391-394 (1991).

13. W. A. de Rooij and C. C. A. H. van der Stap, "Expansion of Mie scattering matrices in generalized spherical functions," Astron. Astrophys. 131, 237-248 (1984).

14. The cross section derived from the experimental curve from the zero angular tilt is $28 \%$ smaller than that calculated from Mie theory. This is likely due to miscalibration of the turbid media concentrations during preparation.

15. F. Reif, Fundamentals of Statistical and Thermal Physics (McGraw-Hill, New York, 1965). 